\title{
Commentaire
}

\section{Consommation problématique de substances ou politiques problématiques en la matière?}

\author{
Tim Stockwell, Ph. D. (1,2); Cecilia Benoit, Ph. D. (1,3); Kiffer Card, Ph. D. (1); Adam Sherk, Ph. D. (1)
}

Diffuser cet article sur Twitter

\section{Résumé}

Ce numéro spécial sur les problèmes de consommation de substances paraît dans une période cruciale pour les responsables des politiques et pour les chercheurs canadiens en matière de santé. À l'heure actuelle, ce sont la crise des opioïdes et les répercussions possibles de la légalisation du cannabis qui retiennent le plus l'attention. Cependant, les substances les plus consommées et les plus nocives demeurent l'alcool et la nicotine. Les politiques visant à réduire les méfaits de ces substances ne suffisent pas : les politiques sur le contrôle de l'alcool sont progressivement abandonnées et on ne saisit pas les occasions de maximiser le potentiel de réduction des méfaits associé aux nouveaux dispositifs d'administration de nicotine plus sécuritaires. Plus généralement, il faut davantage cibler les stratégies de réduction des méfaits fondées sur l'expérience des personnes marginalisées ayant de graves problèmes de consommation de substances, de manière à ne pas aggraver les inégalités en matière de santé.

Afin de mieux éclairer les interventions stratégiques, nous recommandons des approches novatrices de contrôle et de surveillance qui maximisent le recours à de multiples sources de données, comme celles utilisées dans le cadre du projet Coûts et méfaits de l'usage de substances au Canada (CEMUSC). Il faut également accorder une plus grande attention à la précision des définitions de ce qu'est une habitude de consommation à risque et un méfait, afin de favoriser l'adoption de politiques qui tiennent mieux compte des taux réels de méfaits liés à la consommation de substances dans la société canadienne et qui sont davantage capables de les contrer.

Mots-clés : consommation de substances, alcool, cannabis, tabac, tabagisme, réduction des méfaits, surveillance, enquêtes, santé publique, inégalités en matière de santé

\section{Introduction}

On estime que la consommation de substances coûte 46 milliards de dollars par année à l'économie canadienne en dépenses directes en soins de santé, en perte de productivité et en dépenses liées à l'application du code criminel. La grande majorité de ces coûts découle du tabagisme et de la consommation d'alcool ${ }^{1}$. La Stratégie canadienne sur les drogues et autres substances (SCDAS) constitue la réponse officielle du gouvernement fédéral à ces méfaits sociétaux et à ces coûts croissants. Elle est axée sur l'optimisation de quatre activités, soit la prévention, le traitement, la réduction des méfaits et l'application de la loi, grâce à un financement accru de la recherche et des programmes, mais elle cible surtout le cannabis, les opioïdes et d'autres substances illicites.

En 2018, Santé Canada a effectué une évaluation générale de la stratégie du gouvernement fédéral, ce qui a révélé de nombreux problèmes fondamentaux liés à ces quatre activités. Ce numéro spécial sur la consommation problématique de substances au Canada met davantage en
Points saillants

- Au Canada, la consommation de substances a coûté 46 milliards de dollars en 2017, la grande majorité de ces coûts découlant du tabagisme et de la consommation d'alcool.

- Au Canada, les taux de consommation de substances ainsi que les coûts et les méfaits connexes ont augmenté au cours des dernières décennies, que ce soit pour les substances légales ou illégales, en particulier le cannabis

- Les politiques canadiennes en matière de lutte contre la consommation des substances les plus nocives, soit l'alcool, le cannabis et le tabac, sont en grande partie inefficaces, malgré d'importantes possibilités d'amélioration en matière de tarification, de taxation et de commercialisation des substances légales.

- Il est possible d'améliorer les efforts de contrôle et de surveillance au Canada en élaborant des mesures de risque bien pensées et en maximisant l'utilisation de multiples sources de données.

lumière les défis à relever. Outre la conceptualisation de la consommation problématique de nombreuses drogues légales et illégales, ce numéro de la revue Promotion de la santé et prévention des maladies chroniques au Canada offre une description des programmes et des politiques ayant failli à gérer adéquatement les coûts

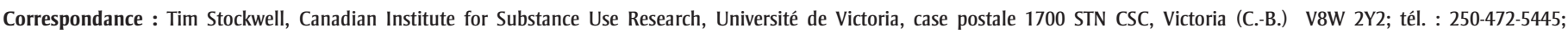
courriel : timstock@uvic.ca 
et les méfaits liés à la consommation de substances.

L'un des points de départ fondamentaux de ce texte est le principe selon lequel les priorités des politiques sur la consommation de substances au Canada doivent intégrer le potentiel de réduction des méfaits connexes. Les politiques doivent en outre s'appuyer sur les expériences des groupes marginalisés afin de ne pas exacerber les inégalités en matière de santé. Nous fournissons des exemples d'orientations stratégiques canadiennes non seulement ayant failli à contrer adéquatement les méfaits du tabagisme et de la consommation d'alcool, mais même les aggravant. En outre, il faut accorder la priorité aux politiques systémiques à large à l'échelle qui portent sur certains déterminants sociaux de la santé à toutes les étapes de la vie, car elles sont susceptibles d'avoir des effets bénéfiques pour tous les types de consommation de substances et de méfaits connexes.

Ce numéro spécial présente huit articles, qui sont axés sur l'évaluation des politiques, la description des tendances actuelles en matière de consommation de substances et l'examen des facteurs de risque. Quatre articles portent sur les politiques en matière d'alcool, trois sur les méfaits de la consommation de cannabis et un sur les facteurs de protection potentiels contre la consommation de substances chez les jeunes. Même si on ne traite pas ici de plusieurs substances importantes et de diverses préoccupations fondamentales (p. ex. la stigmatisation, les populations marginalisées, les déterminants sociaux), ces articles offrent des perspectives pertinentes et actualisées sur certains des plus grands défis auxquels le Canada est actuellement confronté. En effet, la légalisation du cannabis en 2018 a entraîné la réalisation d'une multitude d'études visant à saisir les répercussions sur la prévalence de la consommation de cannabis (en particulier chez les jeunes) ainsi que les futures habitudes de consommation de substances, les méfaits connexes et les effets bénéfiques possibles. En raison de cet intérêt envers le cannabis, les études sur l'alcool et la nicotine ont été reléguées au second plan, bien que ces substances demeurent les plus importantes en termes de coûts et de méfaits.

Les auteurs ont également fourni plusieurs recommandations sur les prochaines étapes à suivre et sur les orientations à envisager, afin d'aider les responsables des politiques et les décideurs. Il s'agit en particulier 1) d'assurer un contrôle et une surveillance continus de la consommation de substances et des méfaits connexes, 2) de mettre en place des mécanismes législatifs et réglementaires visant à réduire les méfaits liés à la consommation de substances (taxes d'accise et prix unitaire minimum des drogues légales, par exemple) et 3) d'accroître la sensibilisation des jeunes, des familles et des collectivités au Canada ainsi que d'améliorer les programmes qui leur sont destinés.

Nous allons traiter de manière plus approfondie ces trois grands thèmes.

\section{Contrôle et surveillance}

Un système de contrôle et de surveillance rigoureux a été mis en place par le gouvernement du Canada, mais il pourrait être amélioré. Le contrôle de la consommation de substances et des méfaits connexes repose sur les enquêtes nationales administrées par Statistique Canada et Santé Canada à ce sujet. Ces enquêtes permettent de brosser le tableau de la consommation de substances, quoiqu'il soit difficile d'en cerner les nouvelles tendances, en raison de retards dans la diffusion des données. Plusieurs programmes, comme le Réseau communautaire canadien d'épidémiologie des toxicomanies, aident à combler ces lacunes en matière de surveillance sentinelle : ils permettent de relier les partenaires locaux à travers le Canada et de cerner les nouvelles tendances et les problèmes potentiels liés à un approvisionnement sécuritaire.

Les enquêtes de Statistique Canada et de Santé Canada permettent des études plus vastes sur la consommation de substances, comme le projet Coûts et méfaits de l'usage de substances au Canada (CEMUSC) qui porte sur le coût économique de la consommation de substances. Le projet CEMUSC compare les coûts et les méfaits en utilisant 8 catégories $^{1}$. En 2017, on a estimé le coût économique global de la consommation de substances - en matière de les soins de santé, de perte de productivité, de justice pénale et d'autres coûts directs - à 46 milliards de dollars. Plus des trois quarts des coûts totaux sont imputables aux trois drogues légales : l'alcool (16,6 milliards de dollars), le tabac $(12,3$ milliards de dollars) et le cannabis (3,2 milliards de dollars). Parmi les substances illicites, les opioïdes (5,9 milliards de dollars) et la cocaïne $(3,7$ milliards de dollars) représentent à eux seuls plus de $5 \%$ du coût total.

On peut classer les efforts de surveillance par ordre de priorité en fonction de deux critères : les méfaits globaux à l'échelle de la population, où l'alcool et le tabac sont de loin les plus importants, et les nouvelles préoccupations importantes en matière de santé, comme la crise des opioïdes.

Plusieurs articles de ce numéro spécial enrichissent les connaissances de référence sur les intoxications au cannabis et sur les méfaits connexes dans les années précédant la légalisation. Selon MaloneyHall et ses collaborateurs ${ }^{2}$, les taux de troubles liés à la consommation de cannabis ont plus que doublé entre 2006 et 2015. Champagne et ses collaborateurs ${ }^{3}$ ont constaté une augmentation des intoxications au cannabis et des méfaits connexes dans la période précédant la légalisation chez les adultes et les jeunes, tandis que Cheng et ses collaborateurs ${ }^{4}$ ont signalé que la majorité des intoxications au cannabis survenaient en concomitance avec la consommation d'alcool. Le contrôle continu des méfaits liés au cannabis, en concomitance avec la consommation d'autres substances, est clairement une priorité depuis la légalisation. Nous recommandons qu'à ce contrôle s'ajoutent des définitions précises concernant les habitudes de consommation de cannabis et les méfaits connexes, notamment la quantification de la consommation. Les méfaits de l'alcool sont pour la plupart liés à la dose, et la consignation des habitudes de consommation uniquement sous l'angle de la fréquence ne suffit pas ${ }^{5}$. Le manque de données concernant la quantité de cannabis consommée par occasion pourrait entraîner une sous-estimation des conséquences sur la santé ${ }^{6}$.

La surveillance de la consommation de substances au Canada devrait demeurer une priorité, et on devrait améliorer la collecte des données. Par exemple, malgré diverses préoccupations, les enquêtes nationales ne permettent pas actuellement de recueillir des renseignements sur la consommation de substances dans les territoires. De plus, les questions des enquêtes devraient permettre de recueillir des renseignements sur la consommation et les méfaits mais aussi sur les nouvelles 
possibilités de réduction des méfaits, comme le remplacement du tabagisme par le vapotage de nicotine ou, possiblement, le remplacement de la consommation d'alcool et d'autres substances par la consommation de cannabis.

\section{Mécanismes législatifs et politiques}

Trois des articles de ce numéro spécial présentent des opportunités d'utilisation, par les gouvernements canadiens, des politiques liées à l'alcool pour améliorer les résultats en matière de santé publique. Selon Paradis et ses collaborateurs ${ }^{7}$, les règlements visant à restreindre le contenu de la promotion de l'alcool dans les médias classiques doivent être étendus aux médias sociaux. En effet, ces auteurs montrent que des bars fréquentés par des étudiants dans quatre villes canadiennes autorisent souvent sur leur compte de médias sociaux des publications qui contreviennent aux règlements en vigueur. Stockwell et ses collaborateurs ${ }^{8}$ identifient des politiques de prix et de taxes liées à l'alcool susceptibles de sauver des centaines de vies au Canada et éviter des milliers d'hospitalisations chaque année. Ils estiment également que le fait de ne pas avoir ajusté les taxes d'accise sur l'alcool en fonction de l'inflation au cours des 25 dernières années a coûté environ 11 milliards de dollars au gouvernement fédéral. Selon Sherk ${ }^{9}$, les recettes actuellement perçues par le gouvernement fédéral sont inférieures d'un tiers aux estimations des coûts économiques nationaux générés par l'alcool.

Le projet d'évaluation des politiques canadiennes sur l'alcool (EPCA) ${ }^{10,11}$ brosse un portrait général de la faillite des gouvernements canadiens en matière d'alcool et de santé publique. Dans le cadre du projet EPCA, plus de 200 indicateurs relevant de 11 domaines de politique fondés sur des données probantes ont été évalués : les gouvernements provinciaux et territoriaux n'ont pas obtenu la note de passage en ce qui concerne la mise en œuvre de la plupart des politiques en matière d'alcool. De nombreuses pratiques rigoureuses ont été recensées, mais les gouvernements provinciaux et territoriaux ont tendance à mettre en place des politiques qui aggravent les effets de l'alcool sur la santé publique, par exemple le programme de bière à $1 \$$ (" buck a beer ") et les ventes dans les épiceries (comme le décrivent Myran et ses collaborateurs ${ }^{12}$ ). Van der Maas et ses collaborateurs ${ }^{13}$ ont montré la viabilité et la valeur potentielle qu'aurait l'ajout d'un volet canadien à l'étude internationale de contrôle de l'alcool (IAC) en ce qui concerne la surveillance et l'évaluation longitudinale des politiques en matière d'alcool.

Le principe selon lequel plus une population consomme d'alcool, plus le risque global pour sa santé et sa sécurité augmente est au cœur de l'approche actuelle de santé publique à l'égard des politiques sur l'alcool ${ }^{14}$. Compte tenu des données probantes de plus en plus importantes associées à l'efficacité des politiques de réduction de la consommation d'alcool dans la population, il serait pertinent d'en appliquer les succès aux autres substances légales. Dans ce numéro, trois articles portent sur les tendances ou les taux des méfaits liés au cannabis relevés par les systèmes de surveillance canadiens. Selon Maloney-Hall et ses collaborateurs ${ }^{2}$, les taux d'hospitalisations liées au cannabis ont doublé entre 2006 et 2015. Près de la moitié de ces hospitalisations étaient attribuables à des troubles mentaux et à des troubles du comportement, dont la psychose, dont le taux a triplé. Champagne et ses collaborateurs ${ }^{3}$ ont signalé une augmentation de $30 \%$ des visites aux urgences pour des intoxications ou des méfaits liés au cannabis entre 2015 et 2018. Par ailleurs, les analyses d'enquêtes canadiennes d'autodéclaration de la consommation de substances menées dans le cadre du projet CEMUSC confirment des augmentations parallèles des taux de consommation de cannabis entre 2007 et $2017^{1}$. Ces tendances montrent qu'il faut avoir des politiques sur le cannabis plus efficaces, comme celles qui sont recensées pour l'alcool dans le cadre du projet EPCA, en ce qui concerne la fixation des prix et la taxation, l'accessibilité et les contrôles du marketing.

\section{Sensibilisation accrue des groupes marginalisés et amélioration des programmes qui leur sont destinés}

Afin de réduire les méfaits chez les personnes ayant de graves problèmes de consommation de substances, dont beaucoup sont marginalisées et stigmatisées, il faut adapter les politiques visant l'ensemble de la population, car elles sont actuellement trop rigides. Les programmes de consommation contrôlée d'alcool constituent une approche de réduction des méfaits unique au Canada. Ils ont comme objectif de réduire les méfaits subis par les personnes qui sont en situation précaire de logement, population au sein de laquelle les peuples autochtones sont surreprésentés dans de nombreuses régions du Canada. Dans le cadre de ces programmes, on fournit un logement aux participants et on leur sert régulièrement de l'alcool dans un environnement sécuritaire ${ }^{15}$. Des travaux sont en cours pour déterminer si le remplacement de l'alcool par le cannabis réduit davantage les méfaits au sein de cette population ${ }^{16}$. En ce qui concerne la nicotine, il faut maximiser le potentiel de réduction des méfaits des méthodes de rechange, qui sont plus sûres, afin de freiner davantage la terrible épidémie de maladies pulmonaires liées au tabac. En 2018, les National Academies of Science, Medicine and Engineering des États-Unis ont conclu que les cigarettes électroniques étaient bien moins nocives que les cigarettes ordinaires ${ }^{17}$. Le fait de fumer des feuilles de cannabis séchées, en particulier en association avec le tabagisme, présente également des risques importants de maladies pulmonaires ${ }^{18}$. Les politiques qui restreignent l'accès des gros fumeurs (en particulier des groupes marginalisés et à faible revenu) aux articles de vapotage risquent d'empêcher ces personnes de profiter des énormes avantages que semble avoir cette nouvelle technologie, susceptible de contribuer à réduire les quelque 50000 décès annuels attribuables aux maladies pulmonaires liées au tabagisme ${ }^{1}$.

Les approches locales de réduction des méfaits sont également recommandées pour les femmes enceintes et les mères qui consomment des substances, population au sein de laquelle les femmes autochtones à faible revenu sont surreprésentées. Ces programmes doivent être évalués de façon à que les fournisseurs de services tiennent réellement compte des intérêts et des préoccupations des parents ainsi que du bien-être de leurs enfants à naître et dont ils sont responsables. Selon Benoit et ses collaborateurs $^{19}$, les fournisseurs de services sociaux et de soins de santé, même lorsqu'ils adoptent des principes de réduction des méfaits, ont tendance à considérer comme problématique ou moralement répréhensible toute consommation de substances chez une femme enceinte ou chez une femme qui est mère depuis peu. En revanche, les nouveaux parents, même 
s'ils considèrent l'abstinence comme un idéal, reconnaissent aux femmes l'autonomie de juger par elles-mêmes du risque lié à la consommation de substances. Les participants ont également attiré l'attention sur les facteurs structurels sociaux (situation de logement précaire, insécurité alimentaire, violence, etc.) qui augmentent les méfaits associés à la consommation de substances ${ }^{20}$. Dans le cadre de programmes de réduction des méfaits visant à fournir sans jugement des soins aux femmes marginalisées et à leur famille, il faut mesurer le succès sous plusieurs angles afin d'évaluer la qualité et les effets des soins, d'améliorer les services et d'appliquer ces expériences à l'élaboration de futurs programmes ${ }^{21}$.

L'étude menée par Enns et Orpana ${ }^{22}$ concernant certains facteurs, en particulier la résilience et l'autonomie, susceptibles de protéger les jeunes contre la consommation de substances nocives est un bon moyen de se rappeler que la prévention doit être axée sur les points forts et pas seulement sur les points faibles.

\section{Conclusion}

Selon nous, les politiques actuelles en matière de consommation de substances sont défaillantes, ou du moins elles n'atteignent pas leur plein potentiel, en particulier en ce qui concerne les drogues légales courantes. Le Canada doit non seulement renforcer le contrôle et la surveillance, mais aussi adopter des approches de modélisation comme celles qui sont appliquées dans le cadre du projet national de surveillance CEMUSC, afin d'obtenir des estimations complètes et précises sur les habitudes de consommation de substances et sur les méfaits connexes ${ }^{1}$. Nous devrions étayer nos interventions stratégiques sur l'établissement de stratégies prioritaires touchant les substances les plus nocives, tout en élaborant des stratégies de réduction des méfaits pour les populations marginalisées qui consomment beaucoup. En ce qui concerne les activités de contrôle et de surveillance, nous ne devrions pas confondre la consommation de substances avec ses méfaits connexes : nous avons besoin de définitions et de mesures précises concernant les habitudes de consommation qui causent le plus de méfaits, tout en reconnaissant le droit des citoyens d'avoir accès à des substances psychoactives et de participer au processus de mise en œuvre des stratégies de réduction des méfaits.
Pour terminer, les éditeurs invités souhaitent remercier les auteurs pour le travail exceptionnel qu'ils ont investi dans ces études et ils souhaitent remercier également l'équipe éditoriale de Promotion de la santé et prévention des maladies chroniques au Canada pour la réalisation de ce numéro spécial.

\section{Conflits d'intérêts}

TS, CB, KC et AS étaient les rédacteurs invités de ce numéro de la Revue PSPMC, mais ils se sont soustraits à toutes les décisions éditoriales concernant ce manuscrit.

\section{Avis}

Le contenu de l'article et les points de vue qui y sont exprimés n'engagent que les auteurs et ne correspondent pas nécessairement à ceux du gouvernement du Canada.

\section{Références}

1. Groupe de travail Coûts et méfaits de l'usage de substances au Canada. Coûts et méfaits de l'usage de substances au Canada (2007-2017). Ottawa (Ont.) : Centre canadien sur les dépendances et l'usage de substances; 2020 (à paraître).

2. Maloney-Hall B, Wallingford S, Konefal S, Young M. Troubles psychotiques et consommation de cannabis : évolution des hospitalisations au Canada, 2006-2015. Promotion de la santé et prévention des maladies chroniques au Canada. 2020;40(5/6):197-204. doi: 10.24095/hpcdp.40.5/6.06f.

3. Champagne A, McFaull S, Thompson W, Bang F. Surveillance de niveau supérieur : surveillance par sentinelle des blessures et des intoxications associées au cannabis. Promotion de la santé et prévention des maladies chroniques au Canada. 2020;40(5/6): 205-214. 10.24095/hpcdp.40.5/6.07f.

4. Cheng P, Zargaran A, Rajabali F, Turcotte K, Babul S. Établissement d'une base de référence : description des intoxications au cannabis dans un hôpital pédiatrique canadien avant la légalisation de la consommation de cannabis à des fins récréatives. Promotion de la santé et prévention des maladies chroniques au Canada. 2020;40(5/6):215-223. doi: 10.24095/hpcdp.40.5/6.08f.
5. Zeisser C, Thompson K, Stockwell T, et al. A "standard joint"? The role of quantity in predicting cannabis-related harm. Addict Res Theory. 2012;20(1): 82-92. doi:10.3109/16066359.2011 .569101 .

6. Asbridge M, Duff C, Marsh DC, Erickson PG. Problems with the identification of 'problematic' cannabis use: examining the issues of frequency, quantity, and drug use environment. Eur Addict Res. 2014;20(5): 254-267. doi:10.1159/000360697.

7. Paradis C, Zhao J, Stockwell T. Publications non conformes des bars populaires sur les plateformes de médias sociaux : une invitation à améliorer la réglementation de la publicité sur l'alcool. Promotion de la santé et prévention des maladies chroniques au Canada. 2020;40(5/6): 160-170. doi:10.24095/hpcdp.40.5/6 $.03 f$.

8. Stockwell T, Churchill S, Sherk A, Sorge J, Gruenewald P. Réduction des décès et des hospitalisations dus à l'alcool grâce à des politiques fiscales et d'établissement des prix différentes? Modélisation des effets sur la consommation d'alcool, les revenus et les méfaits liés à l'alcool au Canada. Promotion de la santé et prévention des maladies chroniques au Canada. 2020;40(5/6):171-184. doi:10.24095 /hpcdp.40.5/6.04f.

9. Sherk A. Déficit associé à l'alcool : recettes publiques et coûts pour la société associés à l'alcool au Canada. Promotion de la santé et prévention des maladies chroniques au Canada. 2020;40(5/6):156-159. doi:10.24095 /hpcdp.40.5/6.02f.

10. Wettlaufer A, Vallance K, Chow C, et al. Reducing alcohol-related harms and costs in Manitoba: a policy review. Victoria (BC): Canadian Institute for Substance Use Research, University of Victoria; 2019.

11. Stockwell T, Vallance K, Chow C, et al. Reducing alcohol-related harms and costs in British Columbia: a policy review. Victoria (BC): Canadian Institute for Substance Use Research, University of Victoria; 2019. 
12. Myran DT, Chen JT, Bearnot B, Ip M, Giesbrecht N, Rees VW. Alcohol availability across neighborhoods in Ontario following alcohol sales deregulation, 2013-2017. Am J Public Health. 2019;109(6):899-905. doi: 10.2105/AJPH.2019.305014.

13. Van der Maas M, Giesbrecht $N$, Stoduto G, Orpana H, Geneau R, Mann R. Habitudes de consommation, méfaits liés à l'alcool et points de vue sur les politiques : résultats d'un projet pilote de l'étude internationale sur le contrôle de l'alcool (IAC) au Canada. Promotion de la santé et prévention des maladies chroniques au Canada. 2020;40(5/6): 185-196. doi:10.24095/hpcdp.40.5/6 $.05 f$.

14. Sherk A, Stockwell T, Chikritzhs T, et al. Alcohol consumption and the physical availability of take-away alcohol: systematic review and meta-analyses of the days and hours of sale and outlet density. J Stud Alcohol Drugs. 2018;79(1):58-67. doi:10.15288/jsad .2018 .79 .58 .

15. Pauly B, Vallance K, Wettlaufer A, et al. Community managed alcohol programs in Canada: overview of key dimensions and implementation. Drug Alcohol Rev. 2018;37(Suppl 1):S132139. doi:10.1111/dar.12681.

16. Subbaraman MS. Substitution and complementarity of alcohol and cannabis: a review of the literature. Subst Use Misuse. 2016;51(11):1399-1414. doi:10.3109/10826084.2016.1170145.

17. Eaton DL, Alberg AJ, Goniewicz M, et al.; Committee on the Review of the Health Effects of Electronic Nicotine Delivery Systems. Public health consequences of e-cigarettes. Consensus study report for the US Food and Drug Administration. Washington (DC): US National Academies of Science, Medicine and Engineering; 2018.

18. Callaghan RC, Verdichevski M, Fyfe TM, Gatley JM. Does cannabis use increase the risk of developing cancer in humans? Dans : Preedy VR (dir.). Handbook of cannabis and related pathologies: biology, pharmacology and treatment. Chapter e9. Cambridge (MA): Elsevier/Academic Press; 2016. p. e80-100.
19. Benoit C, Stengel C, Marcellus L, et al. Providers' constructions of pregnant and early parenting women who use substances. Sociol Health Illn. 2014;36(2):252-263. doi:10.1111 /1467-9566.12106.

20. Benoit C, Magnus S, Phillips R, Marcellus L, Charbonneau S. Complicating the morality discourse: parents' constructions of problematic substance use. Int $\mathrm{J}$ Equity Health. 2015;14:72. doi:10.1186/s12939-015 -0206-7.

21. Marcellus L, MacKinnon K, Benoit C, Phillips R, Stengel C. Reenvisioning definitions of success for programs supporting pregnant women with problematic substance use. Qual Health Res. 2015;25:500-512. doi:10.1177 /1049732314551058.

22. Enns A, Orpana H. Autonomie, compétence et appartenance sociale et consommation de cannabis et d'alcool chez les jeunes au Canada : une analyse transversale. Promotion de la santé et prévention des maladies chroniques au Canada. 2020;40(5/6): 224-234. doi:10.24095/hpcdp.40.5/6 $.09 f$. 